\title{
On sandwich results for some subclasses of analytic functions involving certain linear operator
}

\author{
Amnah Shammaky \\ Department of Mathematics, Faculty of Science, Jazan University, Jazan, Saudi Arabia
}

Abstract: - The purpose of this paper is to derive some subordination and superordination results for certain normalized analytic functions in the open unit disk, acted upon by Carlson-Shaffer operator. Relevant connections of the results, which are presented in the paper, with various known results are also considered Keywords : - differential subordinations ; differential superordinations ; dominant ; subordinant

\section{INTRODUCTION}

Let $H$ be the class of functions analytic in the open unit disk $\Delta=\{z:|z|<1\}$. Let $H[a, n]$ be the subclass of $H$ consisting of functions of the form

$$
f(z)=a+a_{n} z^{n}+a_{n+1} z^{n+1}+\ldots
$$

Let $A$ be the subclass of $H$ consisting of functions of the form

$f(z)=a+a_{2} z^{2}+\ldots$

and we let

$$
A_{m}=\left\{f \in H, f(z)=z+a_{m+1} z^{m+1}+a_{m+2} z^{m+2}+\ldots\right\} \text {. }
$$

With a view to recalling the principle of subordination between analytic functions, let the functions $f$ and $g$ be analytic in $\Delta$. Then we say that the function $f$ is subordinate to $g$ if there exists a Schwarz function $\omega(z)$, analytic in $\Delta$ with

$$
\omega(0)=0 \text { and }|\omega(z)|<1 \quad(z \in \Delta) \text {, }
$$

such that

We denote this subordination by

$$
f(z)=g(\omega(z)) \quad(z \in \Delta) .
$$

$f \prec g$ or $f(z) \prec g(z) \quad(z \in \Delta)$.

In particular, if the function $\mathrm{g}$ is univalent in $\Delta$, the above subordination is equivalent to

$$
f(0)=g(0) \text { and } f(\Delta) \subset g(\Delta) .
$$

Let $p, h \in H$ and let $\phi(r, s, t ; z): C^{3} \times \Delta \rightarrow C$. If $p$ and $\phi\left(p(z), z p^{\prime}(z), z^{2} p^{\prime \prime}(z) ; z\right)$ are univalent and if $p$ satisfies the second-order superordination

$$
h(z) \prec \phi\left(p(z), z p^{\prime}(z), z^{2} p^{\prime \prime}(z) ; z\right) \text {, }
$$

then $p$ is a solution of the differential superordination (1). (If $f$ subordinate to $F$, then $F$ is called to be superordinate to $f$.) An analytic function $q$ is called a subordinant if $q \prec p$ for all $p$ satisfying (1). An univalent subordinant $\tilde{q}$ that satisfies $q \prec \tilde{q}$ for all subordinants $q$ of (1) is said to be the best subordinant. Recently, Miller and Mocanu [7] obtained conditions on $h, q$ and $\phi$ or which the following implication holds

$$
h(z) \prec \phi\left(p(z), z p^{\prime}(z), z^{2} p^{\prime \prime}(z) ; z\right) \Rightarrow q(z) \prec p(z) .
$$

Using the results of Miller and Mocanu [7], Bulboaca[2] considered certain classes of firstorder differential superordinations as well as superordination-preserving integral operators [3]. Ali et al. [1] have used the results of Bulboaca [2] and obtained sufficient conditions for certain normalized analytic functions $f(z)$ to satisfy

$$
q_{1}(z) \prec \frac{z f^{\prime}(z)}{f(z)} \prec q_{2}(z),
$$


where $q_{1}$ and $q_{2}$ are given univalent functions in $\Delta$ with $q_{1}(0)=1$ and $q_{2}(0)=1$. Shanmugam et al. [9] obtained sufficient conditions for a normalized analytic functions $f(z)$ to satisfy

$$
q_{1}(z) \prec \frac{f(z)}{z f^{\prime}(z)} \prec q_{2}(z),
$$

and

where $q_{1}$ and $q_{2}$ are given univalent functions in $\Delta$ with $q_{1}(0)=1$ and $q_{2}(0)=1$.

While Obradovic and Owa [8] obtained subordination results with the quantity . A detailed investing- ation of starlike functions of complex order and convex functions of complex order using Briot - Bouquet differential subordination technique has been studied very recently by Srivastava and Lashin [10] (see also [11]) .

Let the function $\phi(a, c ; z)$ be given by

$$
\varphi(a, c ; z)=\sum_{n=0}^{\infty} \frac{(a)_{n}}{(c)_{n}} z^{n+1} \quad(c \neq 0,-1,-2, \ldots ; z \in \Delta),
$$

where $(x)_{n}$ is the Pochhammer symbol defined by

$$
(x)_{n}=\left\{\begin{array}{l}
1, \quad n=0 ; \\
x(x+1)(x+2) \ldots(x+n-1), \quad n \in N=\{1,2,3, \ldots\} .
\end{array}\right.
$$

Corresponding to the function $\varphi(a, c ; z)$, Carlson and Shaffer [4] introduced a linear operator $L(a, c)$, which is defined by the following Hadamard product (or convolution):

$$
L(a, c) f(z)=\varphi(a, c ; z) * f(z)=\sum_{n=0}^{\infty} \frac{(a)_{n}}{(c)_{n}} a_{n} z^{n+1} .
$$

We note that

$$
L(a, a) f(z)=f(z), \quad L(2,1) f(z)=z f^{\prime}(z), \quad L(\delta+1,1) f(z)=D^{\delta} f(z),
$$

where $D^{\delta} f(z)$ is the Ruscheweyh derivative of $f(z)$.

The main object of the present sequel to the aforementioned works is to apply a method based on the differential subordination in order to derive several subordination results involv- ing the Carlson-Shaffer Operator. Furthermore, we obtain the previous results of Srivastava

and Lashin [10] and Obradović and Owa [8] as special cases of some of the results presented here.

$$
q_{1}(z) \prec \frac{z^{2} f^{\prime}(z)}{\{f(z)\}^{2}} \prec q_{2}(z),
$$

\section{PRELIMINARIES}

In order to prove our subordination and superordination results, we make use of the following known results.

Definition 2.1 [7 Definition 2, p. 817] Denote by $Q$ the set of all functions $f(z)$ that are analytic and injective on $\bar{\Delta}-E(f)$, where

$$
E(f)=\left\{\zeta \in \partial \Delta: \lim _{z \rightarrow \zeta} f(z)=\infty\right\},
$$

and are such that $f^{\prime}(\zeta) \neq 0$ for $\zeta \in \partial \Delta-E(f)$.

Theorem 2.2[6,Theorem 3.4h, p.132] Let the function $q$ be univalent in the open unit disk $\Delta$ and $\theta$ and $\phi$ be analytic in a domain $D$ containing $q(\Delta)$ with $\phi(\omega) \neq 0$ when $\omega \in q(\Delta)$. Set $Q(z)=z q^{\prime}(z) \phi(q(z))$, $h(z)=\theta(q(z))+Q(z)$. Suppose that

(1) $Q(z)$ is starlike univalent in $\Delta$, and 
(2) $\operatorname{Re}\left(z h^{\prime}(z) / Q(z)\right)>0$ for $(z \in \Delta)$.

If

$\theta\{p(z)\}+z p^{\prime}(z) \phi(p(z)) \prec \theta\{q(z)\}+z q^{\prime}(z) \phi(q(z))$,

then $p(z) \prec q(z)$ and $q$ is best dominant.

Lemma $2.3[10]$ Let $g$ be a convex function in $\Delta$ and let

$h(z)=g(z)+m \alpha z g^{\prime}(z)$,

where $\alpha>0$ and $m$ is a positive integer.

If $p(z)=g(0)+p_{m} z^{m}+\ldots$ is analytic in $\Delta$ and

$p(z)+\alpha z p^{\prime}(z) \prec h(z), \quad(z \in \Delta)$,

then

$p(z) \prec g(z), \quad(z \in \Delta)$

and this result is sharp.

Lemma 2.4 [9, Lemma 1, p,71] Let $h$ be a convex function with $h(0)=a$ and let $\gamma \in C$ with $\operatorname{Re}(\gamma) \geq 0$. If $p \in H$ with $p(0)=a$ and

$$
p(z)+\frac{1}{\gamma} z p^{\prime}(z) \prec h(z), \quad(z \in \Delta),
$$

then

$p(z) \prec q(z) \prec h(z)(z \in \Delta)$

where

$q(z)=\frac{\gamma}{n z^{(\gamma / n)}} \int_{0}^{z} h(t) t^{(\gamma / n)^{-1}} d t, \quad(z \in \Delta)$.

The function $q$ is convex and is the best dominant .

Theorem 2.5 [2] Let the function $q$ be convex univalent in the open unit disk $\Delta$ and $\vartheta$ and $\varphi$ be analytic in a domain $D$ containing $q(\Delta)$. Suppose that

(1) $\operatorname{Re}\left[\vartheta^{\prime}(q(z)) / \varphi(q(z))\right]>0$ for $(z \in \Delta)$,

(2) $z q^{\prime}(z) \varphi(q(z))$ is starlike univalent in $\Delta$.

If $p \in H[q(0), 1] \cap Q$, with $p(\Delta) \subseteq D$, and $\vartheta(p(z))+z p^{\prime}(z) \varphi(p(z))$ is univalent in $\Delta$, and

$$
\vartheta\{q(z)\}+z q^{\prime}(z) \varphi(q(z)) \prec \vartheta\{p(z)\}+z p^{\prime}(z) \varphi(p(z)),
$$

then $q(z) \prec p(z)$ and $q$ is the best subordinant .

\section{SUBORDINATION AND SUPERORDINATION FOR ANALYTIC FUNCTIONS} We begin by proving involving differential subordination between analytic functions .

Lemma3.1 If $f(z) \in \Delta$, then $z(L(a, c) f(z))^{\prime}=a L(a+1, c) f(z)-(a-1) L(a, c) f(z)$, where $c \neq 0,-1,-2, \ldots$

proof Note that

$$
L(a, c) f(z)=\sum_{n=0}^{\infty} \frac{(a)_{n}}{(c)_{n}} a_{n} z^{n+1},
$$

and

$$
L(a+1, c) f(z)=\sum_{n=0}^{\infty} \frac{(a+1)_{n}}{(c)_{n}} a_{n} z^{n+1} .
$$

These give that 


$$
\begin{aligned}
& a L(a+1, c) f(z)-(a-1) L(a, c) f(z) \\
& =\sum_{n=0}^{\infty}(a+n) \frac{(a)_{n}}{(c)_{n}} a_{n} z^{n+1}-\sum_{n=0}^{\infty}(a-1) \frac{(a)_{n}}{(c)_{n}} a_{n} z^{n+1} \\
& =\sum_{n=0}^{\infty}(n+1) \frac{(a)_{n}}{(c)_{n}} a_{n} z^{n+1} \\
& =z(L(a, c) f(z))^{\prime}
\end{aligned}
$$

this proves lemma 3.1 .

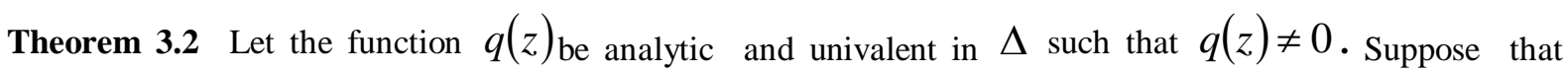
$z q^{\prime}(z) / q(z)$ is starlike univalent in $\Delta$. Let

$$
\operatorname{Re}\left\{1+\frac{\xi}{\beta} q(z)+\frac{2 \delta}{\beta}(q(z))^{2}-\frac{z q^{\prime}(z)}{q(z)}+\frac{z q^{\prime \prime}(z)}{q^{\prime}(z)}\right\}>0 \quad(z \in \Delta ; \alpha, \delta, \xi, \beta \in C ; \beta \neq 0)
$$

and

$$
\psi(a, c, \mu, \xi, \beta, \delta, \eta, f)=\left\{\begin{array}{l}
\alpha+\xi\left(\frac{L(a, c) f(z)}{z}\right)^{\mu}\left(\frac{z}{L(a+1, c) f(z)}\right)^{\eta} \\
+\delta\left(\frac{L(a, c) f(z)}{z}\right)^{2 \mu}\left(\frac{z}{L(a+1, c) f(z)}\right)^{2 \eta} \\
+\beta \mu(a)\left[\frac{L(a+1, c) f(z)}{L(a, c) f(z)}-1\right] \\
+\beta \eta(1+a)\left[1-\frac{L(a+2, c) f(z)}{L(a+1, c) f(z)}\right]
\end{array}\right\}
$$

If $q$ satisfies the following subordination :

$$
\begin{gathered}
\psi(a, c, \mu, \xi, \beta, \delta, \eta, f) \prec \alpha+\xi q(z)+\delta(q(z))^{2}+\beta \frac{z q^{\prime}(z)}{q(z)} \\
(z \in \Delta ; \alpha, \delta, \xi, \beta, \mu, \eta \in C ; \mu \neq 0 ; \beta \neq 0),
\end{gathered}
$$

then

$$
\left(\frac{L(a, c) f(z)}{z}\right)^{\mu}\left(\frac{z}{L(a+1, c) f(z)}\right)^{\eta} \prec q(z) \quad(z \in \Delta ; z \neq 0 ; \mu, \eta \in C ; \mu \neq 0)
$$

and $q$ is the best dominant .

Proof Let the function $p(z)$ be defined by

$$
p(z)=\left(\frac{L(a, c) f(z)}{z}\right)^{\mu}\left(\frac{z}{L(a+1, c) f(z)}\right)^{\eta}
$$

so that, by a straightforward computation, we have

$$
\frac{z p^{\prime}(z)}{p(z)}=\mu\left[\frac{z(L(a, c) f(z))^{\prime}}{L(a, c) f(z)}-1\right]+\eta\left[1-\frac{z(L(a+1, c) f(z))^{\prime}}{L(a+1, c) f(z)}\right] .
$$

By using lemma3.1we deduce that 


$$
\frac{z p^{\prime}(z)}{p(z)}=\mu\left[\frac{a L(a+1, c) f(z)}{L(a, c) f(z)}-a\right]+\eta\left[(1+a)-\frac{(1+a) L(a+2, c) f(z)}{L(a+1, c) f(z)}\right] .
$$

By setting

$$
\theta(\omega)=\alpha+\xi \omega+\delta \omega^{2} \text { and } \phi(\omega)=\frac{\beta}{\omega}
$$

it can be easily observed that $\theta(\omega)$ is analytic in $C, \phi(\omega)$ is analytic in $C \backslash\{0\}$ and that $\phi(\omega) \neq 0 \quad(\omega \in C \backslash\{0\})$.

Also, by letting

$$
Q(z)=z q^{\prime}(z) \phi(q(z))=\beta \frac{z q^{\prime}(z)}{q(z)}
$$

and

$$
h(z)=\theta\{q(z)\}+Q(z)=\alpha+\xi q(z)+\delta(q(z))^{2}+\beta \frac{z q^{\prime}(z)}{q(z)}
$$

we find that $Q(z)$ is starlike univalent in $\Delta$ and that

$$
\begin{gathered}
\operatorname{Re}\left(\frac{z h^{\prime}(z)}{Q(z)}\right)=\operatorname{Re}\left\{1+\frac{\xi}{\beta} q(z)+\frac{2 \delta}{\beta}(q(z))^{2}-\frac{z q^{\prime}(z)}{q(z)}+\frac{z q^{\prime \prime}(z)}{q^{\prime}(z)}\right\}>0 \\
(z \in \Delta ; \alpha, \delta, \xi, \beta \in C ; \beta \neq 0) .
\end{gathered}
$$

The assertion (6) of Theorem 3.2 now follows by an application of Theorem 2.2.

$$
\text { For the choices } q(z)=1+A z / 1+B z,-1 \leq B<A \leq 1
$$

and

$q(z)=\{1+z / 1-z\}^{\gamma}, 0<\gamma \leq 1$, in Theorem 3.2, we get the following results (Corollaries 3.3 and 3.4 ).

Corollary 3.3 Assume that (3) holds. If $f \in A$, and

$$
\psi(a, c, \mu, \xi, \beta, \delta, \eta, f) \prec \alpha+\xi \frac{1+A z}{1+B z}+\delta\left(\frac{1+A z}{1+B z}\right)^{2}+\frac{\beta(A-B) z}{(1+A z)(1+B z)}
$$

$$
(z \in \Delta ; \alpha, \delta, \xi, \beta, \mu, \eta \in C ; \mu \neq 0 ; \beta \neq 0),
$$

where $\quad \psi(a, c, \mu, \xi, \beta, \delta, \eta, f) \quad$ is $\quad$ as $\quad$ defined then $\left(\frac{L(a, c) f(z)}{z}\right)^{\mu}\left(\frac{z}{L(a+1, c) f(z)}\right)^{\eta} \prec \frac{1+A z}{1+B z} \quad(z \in \Delta ; z \neq 0 ; \mu, \eta \in C ; \mu \neq 0)$ and $1+A z / 1+B z$ is the best dominant.

Corollary3. 4 Assume that (3) holds. If $f \in A$, and

$$
\begin{gathered}
\psi(a, c, \mu, \xi, \beta, \delta, \eta, f) \prec \alpha+\xi\left(\frac{1+z}{1-z}\right)^{\gamma}+\delta\left(\frac{1+z}{1-z}\right)^{2 \gamma}+\frac{2 \beta \gamma z}{\left(1-z^{2}\right)} \\
(z \in \Delta ; \alpha, \delta, \xi, \beta, \mu, \eta \in C ; \mu \neq 0 ; \beta \neq 0)
\end{gathered}
$$

where $\psi(a, c, \mu, \xi, \beta, \delta, \eta, f)$ is as defined in (4), then $\left(\frac{L(a, c) f(z)}{z}\right)^{\mu}\left(\frac{z}{L(a+1, c) f(z)}\right)^{\eta} \prec\left\{\frac{1+z}{1-z}\right\}^{\gamma} \quad(z \in \Delta ; z \neq 0 ; \mu, \eta \in C ; \mu \neq 0)$ 
and $\{1+z / 1-z\}^{\gamma}$ is the best dominant.

For a special case $q(z)=e^{\mu A z}$, with $|\mu A|<\pi$, Theorem 3.2 readily yields the following.

Corollary3.5 Assume that (3) holds. If $f \in A$, and

$$
\begin{gathered}
\psi(a, c, \mu, \xi, \beta, \delta, \eta, f) \prec \alpha+\xi e^{\mu A z}+\delta e^{2 \mu A z}+\beta A \mu z \\
(z \in \Delta ; \alpha, \delta, \xi, \beta, \mu, \eta \in C ; \mu \neq 0 ; \beta \neq 0)
\end{gathered}
$$

where $\psi(a, c, \mu, \xi, \beta, \delta, \eta, f)$ is as defined in (4), then

$\left(\frac{L(a, c) f(z)}{z}\right)^{\mu}\left(\frac{z}{L(a+1, c) f(z)}\right)^{\eta} \prec e^{\mu A z} \quad(z \in \Delta ; z \neq 0 ; \mu, \eta \in C ; \mu \neq 0)$

and $e^{\mu A z}$ is the best dominant .

Remark 3.6 Taking $a=c=1, \delta=\xi=\eta=0, \alpha=1, \beta=1 / \mu$ in corollary 3.5, we get the

result obtained by Obradovic and Owa [8].

For a special case

when $q(z)=1 /(1-z)^{2 b}(b \in C \backslash\{0\}), a=c=1, \delta=\xi=\eta=0, \alpha=1, \mu=\alpha=1$ and $\beta=1 / b$,

Theorem 3.2 reduces at once to the following known result obtained by Srivastava and Lashin [10].

Corollary3. 7 Let be a non-zero complex number. If $f \in A$, and

$1+\frac{1}{b}\left[\frac{z f^{\prime}(z)}{f(z)}-1\right] \prec \frac{1+z}{1-z}$

then

$$
\frac{f(z)}{z} \prec \frac{1}{(1-z)^{2 b}}
$$

and $1 /(1-z)^{2 b}$ is the best dominant .

For $q(z)=(1+B z)^{\mu(A-B) / B}, a=c=1, \delta=\xi=\eta=0, \alpha=1, \beta=1 / \mu$ in Theorem 3.2, we get the following known result obtained by Obradovic and Owa [8].

Corollary 3.8 Let $-1 \leq B<A \leq 1$. Let $\mu, A, B$ satisfy the relation either

$$
|\mu(A-B) / B-1| \leq 1 \quad \text { or }|\mu(A-B) / B+1| \leq 1 \text {. If } f \in A \text {, and }
$$

then

$$
\frac{z f^{\prime}(z)}{f(z)} \prec \frac{1+A z}{1+B z}
$$

$$
\left(\frac{f(z)}{z}\right)^{\mu} \prec(1+B z)^{\mu(A-B) / B} \quad(z \in \Delta ; z \neq 0 ; \mu \in C ; \mu \neq 0)
$$

and $(1+B z)^{\mu(A-B) / B} \quad$ is the best dominant.

With the help of Lemma 2.4, we now prove the following theorem .

Theorem 3.9 Let $h \in H, h(0)=1, h^{\prime}(0) \neq 0$ which satisfy the inequality

$$
\operatorname{Re}\left[1+\frac{z h^{\prime \prime}(z)}{h^{\prime}(z)}\right]>-\frac{1}{2}, \quad(z \in \Delta) \text {. }
$$

If $f \in A_{m}$ satisfies the differential subordination 


$$
\frac{L(a+k, c) f(z)}{z} \prec h(z), \quad\left(k \in Z^{+}, z \in \Delta ; z \neq 0\right),
$$

then

$$
\frac{L(a+k-1, c) f(z)}{z} \prec g(z), \quad\left(k \in Z^{+}, z \in \Delta ; z \neq 0\right),
$$

where

$$
g(z)=\frac{(a+k-1)}{m z^{((a+k-1) / m)}} \int_{0}^{z} h(t) t^{((a+k-1) / m)^{-1}} d t, \quad\left(k \in Z^{+}, z \in \Delta\right) .
$$

The function $g$ is convex and is the best dominant .

Proof Let the function $p(z)$ be defined by

$$
p(z)=\frac{L(a+k-1, c) f(z)}{z} \quad\left(k \in Z^{+}, z \in \Delta ; z \neq 0\right) .
$$

A straightforward computation gives

By using lemma3.1we deduce that

$$
\frac{z p^{\prime}(z)}{p(z)}=\left[\frac{z(L(a+k-1, c) f(z))^{\prime}}{L(a+k-1, c) f(z)}-1\right] .
$$

$$
\frac{z p^{\prime}(z)}{p(z)}=\left[\frac{(a+k-1) L(a+k, c) f(z)}{L(a+k-1, c) f(z)}-(a+k-1)\right]
$$

and hence

$$
p(z)+\frac{z p^{\prime}(z)}{a+k-1}=\frac{L(a+k, c) f(z)}{z} \quad\left(k \in Z^{+}, z \in \Delta\right) .
$$

The assertion of Theorem 3.9 now follows from Lemma 2.4.

For the choice of $\mathrm{k}=1$, we get

Theorem 3.10 If $f \in A_{m}$ satisfies the differential subordination

$$
\frac{L(a+1, c) f(z)}{z} \prec h(z), \quad(z \in \Delta ; z \neq 0),
$$

then

$$
\frac{L(a, c) f(z)}{z} \prec g(z), \quad(z \in \Delta ; z \neq 0),
$$

where

$$
g(z)=\frac{(1+a)}{m z^{(a / m)}} \int_{0}^{z} h(t) t^{(a / m)^{-1}} d t, \quad(z \in \Delta) .
$$

The function $g$ is convex and is the best dominant .

Proof Let the function $p(z)$ be defined by

$$
p(z)=\frac{L(a, c) f(z)}{z} \quad(z \in \Delta ; z \neq 0),
$$

so that, by a straightforward computation, we have

$$
\frac{z p^{\prime}(z)}{p(z)}=\left[\frac{z(L(a, c) f(z))^{\prime}}{L(a, c) f(z)}-1\right] .
$$

By using lemma3.1we deduce that 


$$
\frac{z p^{\prime}(z)}{p(z)}=\left[\frac{a L(a+1, c) f(z)}{L(a, c) f(z)}-a\right]
$$

and hence

$$
p(z)+\frac{z p^{\prime}(z)}{a}=\frac{L(a+1, c) f(z)}{z} \quad(z \in \Delta) .
$$

The assertion of Theorem 3.10 now follows from Lemma 2.4 .

Next, by using Lemma 2.3, we prove the following theorem .

Theorem 3.11 Let $g$ be a convex function with $g(0)=1$. Let $h$ be a function, such that

$$
h(z)=g(z)+\frac{m}{\lambda+1} z g^{\prime}(z) \text {. }
$$

If $f \in A_{m}$ satisfies the differential subordination

$$
\frac{L(a+k, c) f(z)}{z} \prec h(z),\left(k \in Z^{+}, z \in \Delta ; z \neq 0\right),
$$

then

$$
\frac{L(a+k-1, c) f(z)}{z} \prec g(z), \quad\left(k \in Z^{+}, z \in \Delta ; z \neq 0\right),
$$

and this result is sharp.

Proof The proof of the theorem is much akin to the proof of Theorem 3.12 and hence we omit the details involved.

Next, by appealing to Theorem 2.5 of the preceding section, we prove Theorem 3.12.

Theorem 3.12 1 Let $q(z)$ be analytic and convex univalent in $\Delta$ such that $q(z) \neq 0$ and $z q^{\prime}(z) / q(z)$ be starlike univalent in $\Delta$. Further, let us assume that

$$
\begin{aligned}
& \operatorname{Re}\left\{\frac{2 \delta}{\beta}(q(z))^{2}+\frac{\xi}{\beta} q(z)\right\}>0,(z \in \Delta ; \delta, \xi, \beta \in C ; \beta \neq 0) \\
& \text { If } \quad f \in A, 0 \neq\left(\frac{L(a, c) f(z)}{z}\right)^{\mu}\left(\frac{z}{L(a+1, c) f(z)}\right)^{\eta} \in H[q(0), 1] \cap Q,
\end{aligned}
$$

$\psi(a, c, \mu, \xi, \beta, \delta, \eta, f)$ is univalent in $\Delta$, then

$$
\begin{gathered}
\alpha+\xi q(z)+\delta(q(z))^{2}+\beta \frac{z q^{\prime}(z)}{q(z)} \prec \psi(a, c, \mu, \xi, \beta, \delta, \eta, f) \\
(z \in \Delta ; \alpha, \delta, \xi, \beta, \mu, \eta \in C ; \mu \neq 0 ; \beta \neq 0),
\end{gathered}
$$

implies

$$
q(z) \prec\left(\frac{L(a, c) f(z)}{z}\right)^{\mu}\left(\frac{z}{L(a+1, c) f(z)}\right)^{\eta} \in H[q(0), 1] \cap Q
$$

and $q$ is the best subordinant where $\psi(a, c, \mu, \xi, \beta, \delta, \eta, f)$ is as defined in (4).

Proof By setting

$$
\vartheta(\omega)=\alpha+\xi \omega+\delta \omega^{2} \text { and } \quad \varphi(\omega)=\frac{\omega^{\prime}}{\omega},
$$

it is easily observed that $\vartheta(\omega)$ is analytic in $C$. Also, $\varphi(\omega)$ is analytic in $C \backslash\{0\}$ and that

$$
\varphi(\omega) \neq 0, \quad(\omega \in C \backslash\{0\}) .
$$


Since $q$ is convex (univalent) function it follows that,

$$
\operatorname{Re}\left[\frac{\vartheta^{\prime}(q(z))}{\varphi(q(z))}\right]=\operatorname{Re}\left\{\frac{2 \delta}{\beta}(q(z))^{2}+\frac{\xi}{\beta} q(z)\right\}>0,(z \in \Delta ; \delta, \xi, \beta \in C ; \beta \neq 0) .
$$

The assertion (26) of Theorem 3.12 follows by an application of Theorem 2.5.

We remark here that Theorem 3.12 can easily be restated, for different choice of the function $q(z)$. Combining Theorem 3.2 and Theorem 3.12, we get the following sandwich theorem .

Theorem 3.13 Let $q_{1}(z)$ be convex univalent and $q_{2}(z)$ be univalent in $\Delta$ such that $q_{1}(z) \neq 0$ and $q_{2}(z) \neq 0$. Suppose $f$ satisfies (25)and (3).

If $f \in A, 0 \neq\left(\frac{L(a, c) f(z)}{z}\right)^{\mu}\left(\frac{z}{L(a+1, c) f(z)}\right)^{\eta} \in H[q(0), 1] \cap Q$,

and that $\psi(a, c, \mu, \xi, \beta, \delta, \eta, f)$ is univalent in $\Delta$, where $\psi(a, c, \mu, \xi, \beta, \delta, \eta, f)$ is given by (4) then

$\alpha+\xi q_{1}(z)+\delta\left(q_{1}(z)\right)^{2}+\beta \frac{z q_{1}^{\prime}(z)}{q_{1}(z)} \prec \psi(a, c, \mu, \xi, \beta, \delta, \eta, f) \prec \alpha+\xi q_{2}(z)+\delta\left(q_{2}(z)\right)^{2}+$

$\beta \frac{z q_{2}^{\prime}(z)}{q_{2}(z)}(z \in \Delta ; \alpha, \delta, \xi, \beta, \mu, \eta \in C ; \mu \neq 0 ; \beta \neq 0)$,

implies

$q_{1}(z) \prec\left(\frac{L(a, c) f(z)}{z}\right)^{\mu}\left(\frac{z}{L(a+1, c) f(z)}\right)^{\eta} \prec q_{2}(z) \quad(z \in \Delta ; z \neq 0 ; \mu, \eta \in C ; \mu \neq 0)$ and $q_{1}$ and

$q_{2}$ are respectively the best subordinant and best dominant.

\section{REFERENCES}

[1] R.M. Ali, V. Ravichandran, M. Hussain Khan, and K.G. Subramanian, Differential sandwich theorems for certain analytic functions, Far East J. Math. Sci. 15(1) (2005), pp. 87-94

[2] T. Bulboaca, Classes of first-order differential superordinations, Demonstratio Math. 35(2) (2002), pp. 287-292.

[3] T. Bulboaca, A class of superordination-preserving integral operators, Indag. Math. (N. S.) 13(3) (2002),pp. 301-311.

[4] B.C. Carlson and D.B. Shaffer, Starlike and prestarlike hypergeometric functions, SIAM J. Math. Anal. 15 (1984), pp. 737-745.

[5] S.S. Miller and P.T. Mocanu, On some classes of first order differential subordinations, Mi-chigan Math. J. 32 (1985), pp. 185-195.

[6] S.S. Miller and P.T. Mocanu, Differential subordinations: theory and applications, in Pure and Applied Mathematics No. 225, Marcel Dekker, New York, 2000.

[7] S.S. Miller and P.T. Mocanu, Subordinants of differential superordinations, Complex Var. 48(10) (2003), pp. 815-826.

[8] M. Obradovicc and S. Owa, On certain properties for some classes of starlike functions, J. Math. Anal. Appl. 145(2) (1990), pp. 357364.

[9] T.N. Shanmugam, V. Ravichandran, and S. Sivasubramanian, Differential sandwich theo- rems for some subclasses of analytic functions, Aust. J. Math. Anal. Appl. 3(1) (2006), Article 8, 11

[10] H.M. Srivastava and A.Y. Lashin, Some applications of the Briot-Bouquet differential subordination, JIPAM. J. Inequal. Pure Appl. Math. 6(2) (2005), Article 41, 7.

[11] N. Tuneski, On certain sufficient conditions for starlikeness, Int. J. Math. Math. Sci. 23(8) (2000), pp. 521-527. 